\title{
HIGH RESOLUTION GEODETIC EARTH OBSERVATION WITH TERRASAR-X: CORRECTION SCHEMES AND VALIDATION
}

\author{
Ulrich Balss ${ }^{1}$, Christoph Gisinger ${ }^{2}$, Xiao Ying Cong ${ }^{3}$, Ramon Brcic ${ }^{1}$, Peter Steigenberger ${ }^{2}$, Michael \\ Eineder $^{1,3}$, Roland Pail ${ }^{2}$, and Urs Hugentobler ${ }^{2}$ \\ ${ }^{1}$ Remote Sensing Technology Institute (IMF), German Aerospace Center (DLR), \\ D-82230 Oberpfaffenhofen, Germany
}
${ }^{2}$ Institute for Astronomical and Physical Geodesy (IAPG), Technische Universität München (TUM), D-80333 Munich, Germany
${ }^{3}$ Remote Sensing Technology (LMF), Technische Universität München (TUM), D-80333 Munich, Germany

\begin{abstract}
Previous studies have shown the unprecedented absolute pixel localization accuracy of the German SAR (Synthetic Aperture Radar) satellites TerraSAR-X and TanDEM-X. Now, by thoroughly correcting all signal path delays and geodynamic effects like tides, loadings and plate movements, range accuracies of about 1 centimeter are demonstrated to be attainable. While Global Navigation Satellite System (GNSS) data provide local correction values for the atmospheric delays, correction values for the geodynamic effects are based on the IERS (International Earth Rotation and Reference Systems Service) conventions. Our recent measurements are based on a corner reflector with very precisely known ground position which we installed at Wettzell, Germany, close to the local GNSS reference stations. Further comparable high precision test sites in the world are in progress and shall prove the worldwide reproducibility of the achieved results.
\end{abstract}

Index Terms - Synthetic aperture radar, TerraSAR-X, pixel localization accuracy, imaging geodesy, global navigation satellite system

\section{INTRODUCTION}

The German SAR (Synthetic Aperture Radar) satellites TerraSAR-X (TSX-1) and TanDEM-X (TDX-1), launched in June 2007 and June 2010 respectively, provide an unprecedented geometric accuracy. Previous studies showed an absolute pixel localization accuracy for both sensors at the centimeter level [1-5]. However, we expect that by a thorough correction of all path delays and geodynamic effects even an improvement to the sub-centimeter level seems attainable [6]. As these effects are likewise relevant for the
Global Navigation Satellite System (GNSS), GNSS shall provide a primary data source for the respective correction values.

\section{MEASUREMENT METHOD}

Radar systems indirectly measure geometric distances by means of the two-way travel time of radar pulses from the radar transmitter to the ground and back to the radar receiver. In a focused SAR image, the instant of closest approach of the sensor and target as well as the signal travel time at this instant define the two radar time coordinates of azimuth and range. Usually, the conversion from range time to geometric distance refers to the vacuum velocity of light. However, electrons in the ionosphere, dry air and water vapor in the troposphere introduce additional signal delays which have to be taken into account. In addition, geodynamic effects like tides, loadings and plate movements shift the true position of a ground target.

In order to verify the pixel localization accuracy of a SAR system, the range and azimuth times of corner reflectors in focused SAR images are compared with their expected values obtained from precise on ground measurements of their positions and estimated propagation delays. The conversion of the spatial geodetic coordinates into expected radar time coordinates is based on the zero Doppler equations [7] and interpolation of the satellite's position.

Our recent measurement series is based on a 1.5 meter trihedral corner reflector which we installed at the Geodetic Observatory at Wettzell, Germany. In this way, we benefit from the very close distance (about 240 meters) to the local GNSS reference stations. The ground position of the corner reflector is known very precisely ( $<1$ centimeter) relative to the reference stations as obtained from a terrestrial geodetic survey. 


\section{PATH DELAY CORRECTIONS}

Regarding the impact of the atmosphere on the observed ranges, SAR and GNSS measurements behave very similarly as both make use of radio signals in the Gigahertz range and thus, the concept of separating the atmosphere into troposphere and ionosphere which is well-established in the field of GNSS [8] can be applied the same way to SAR. Moreover, this allows a straight forward transfer of tropospheric and ionospheric signals that are observed by GNSS in terms of Zenith Path Delay (ZPD) and vertical Total Electron Content (vTEC) to the TerraSAR-X range measurements. Since Wettzell station is part of the global IGS (International GNSS Service) GNSS network, all IGS products including the ZPDs and the daily Differential Code Biases (DCBs), which are required for the vTEC computation, are directly available for the individual Wettzell GNSS receivers [9]. The following two procedures were carried out for all Wettzell GNSS receivers available during the datatakes and their mean value yielded the individual tropospheric and ionospheric corrections for the corner reflector measurements.

In order to determine the actual tropospheric corrections, three steps are involved: first, the ZPDs, comprising the total impact of the troposphere, are divided into their hydrostatic and wet components by modeling the hydrostatic part with the equation of Saastamoinen [10]. Next, the two components are individually transferred from the height of the GNSS receivers to the height of the corner reflector using the procedure given in [11]. Finally, the Vienna Mapping Function 1 [11][12] is used to convert both ZPD components into the SAR acquisition geometry.

Like the tropospheric corrections, the corrections for the ionosphere are also solely based on the GNSS measurements at Wettzell. When combined with the ionospheric Single Layer Model (SLM), the description of the ionosphere in terms of vTEC can be obtained from the geometry-free linear combination of dual-frequency GNSS measurements. This computation is possible for every measurement epoch since the usually unknown DCBs are provided by IGS for both the Wettzell receivers and all GNSS satellites. Thus, one obtains the vTEC as a sampled function of time at the Ionospheric Pierce Point (IPP, i.e. the point at which the line of sight intersects with the shell of the SLM) location of the GNSS satellites given by the SLM. By performing a least squares fit of a plane to the sparse GNSS-based vTEC distribution and interpolating the vTEC at the TerraSAR-X IPP, the ionospheric correction for the corner reflector observation can be calculated. The details of the whole procedure can be found in [13].

As the orbits of TSX-1 and TDX-1 are still within the upper ionosphere, the upper portion of the ionosphere contributes to the GNSS based measurement values but not to the path delay of the radar signal. For this reason, we apply a $75 \%$ weighting factor which was derived from an analysis

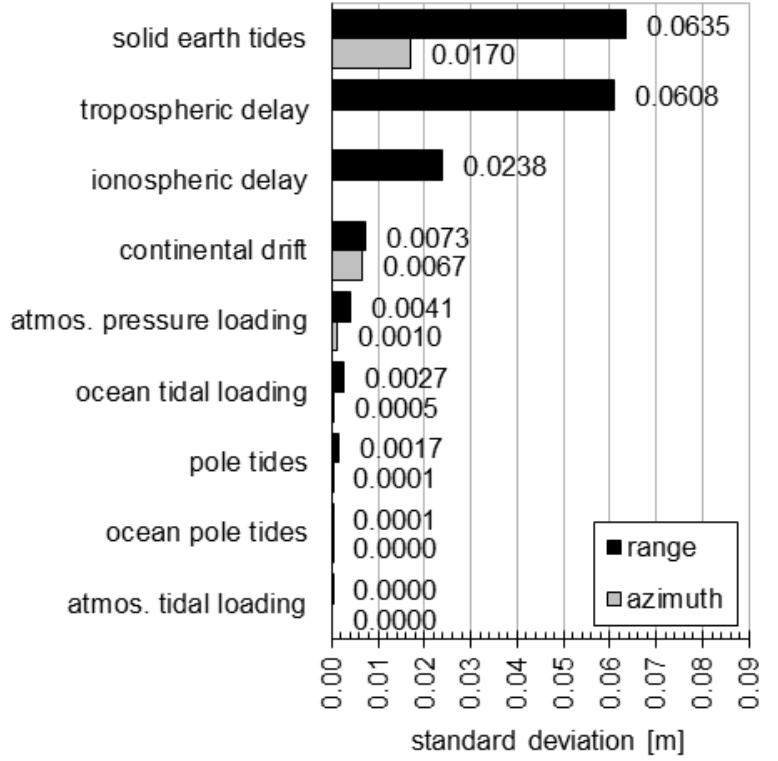

Figure 1: Standard deviations of geodynamic effects and signal path delays

[6] of the IRI-2007 (International Reference Ionosphere) model [14].

\section{CORRECTION OF GEODYNAMIC EFFECTS}

The most prominent geodynamic effects are solid earth tides and continental drift which cause a shift of up to a few decimeters over the course of a day or years, respectively. We already considered both effects in our previous investigations [1][2][5][6]. However, to obtain millimeter localization accuracy, smaller geophysical effects must also be compensated for: Atmospheric pressure loading and ocean tidal loading weigh on the tectonic plate. Their variation shifts the target position by several millimeters each. An even weaker effect (few millimeters) is caused by pole tides. All of these effects can be estimated by state of the art models [15] which are transferred to the TSX-1 acquisition geometry [1]. The correction of these effects follows the conventions issued by IERS (International Earth Rotation and Reference Systems Service) 2010 edition [15]. An exception is atmospheric pressure loading that is not yet included in the IERS conventions but still in debate. Precomputed estimates of this effect for several GNSS reference stations are available from the NASA atmospheric pressure loading service [16][17].

Figure 1 illustrates the proportions of the standard deviations of the individual geodynamic effects and signal path delays based on the example of our Wettzell measurement series. However, the amount of some effects may differ significantly from test site to test site, e.g. ocean tidal loading becomes more relevant for coastal test sites than for an inner land test site like Wettzell. 


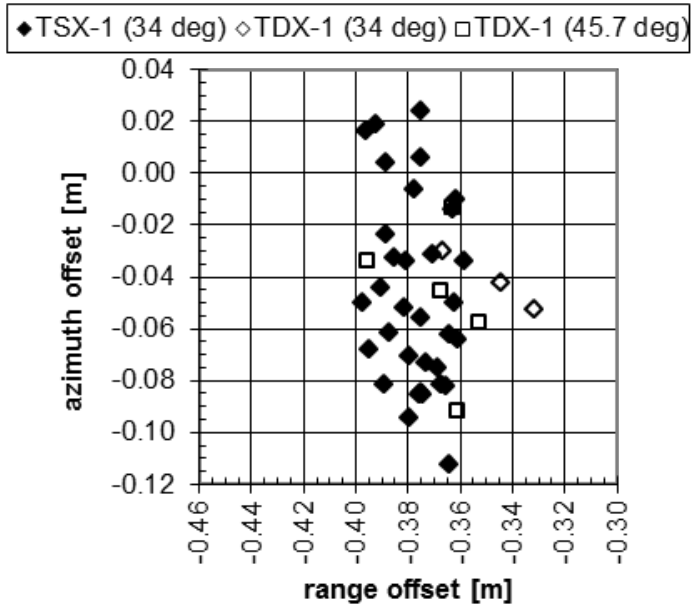

Figure 2: Difference between SAR and GNSS coordinates in radar geometry

\section{MEASUREMENT RESULTS}

Up to now, our measurement series consists of 34 TSX-1 and 5 TDX-1 datatakes of the Wettzell test site which were recorded between July 12, 2011 and May 13, 2013. In case of one TSX-1 and two TDX-1 datatakes in winter 2012/13, a loss in radar cross section of more than $10 \mathrm{~dB}$ (compared to the theoretically derived expected value which is actually reached in the other datatakes) indicates that there was a large amount of snow in the corner reflector which affected the validity of the respective position measurement. For this reason, we have to exclude these 3 datatakes from the analysis below. The predominance of TSX-1 datatakes in the measurement series is due to the fact that in the first stage of our project, we intentionally focused on only one of both sensors (TSX-1) in order to avoid possible unknown systematic differences. The study of such sensor dependencies is the subject of ongoing investigations.

Figure 2 shows the difference between measured and expected radar times after correcting the measured radar times for the signal propagation delays and the geodynamic effects (for convenience the time differences are converted to corresponding spatial distances). The major component of the observed range bias of -37.5 centimeters results from the instrument calibration constants which were determined based on a simplified model for the signal path delays [18] and therefore also contain atmospheric information. This calibration approach suffices for a localization accuracy better than 1 meter as specified in the TerraSAR-X product requirements [19]. In contrast, for high precision localizations as required for the measurement series at hand, an adaptation of the calibration constants is necessary. In the case of the TSX-1 datatakes, the standard deviation of the repeat pass acquisitions amounts to 36.3 millimeters in azimuth and 11.4 millimeters in range. Based on the sparse statistics of the up to now acquired TDX-1 datatakes, the

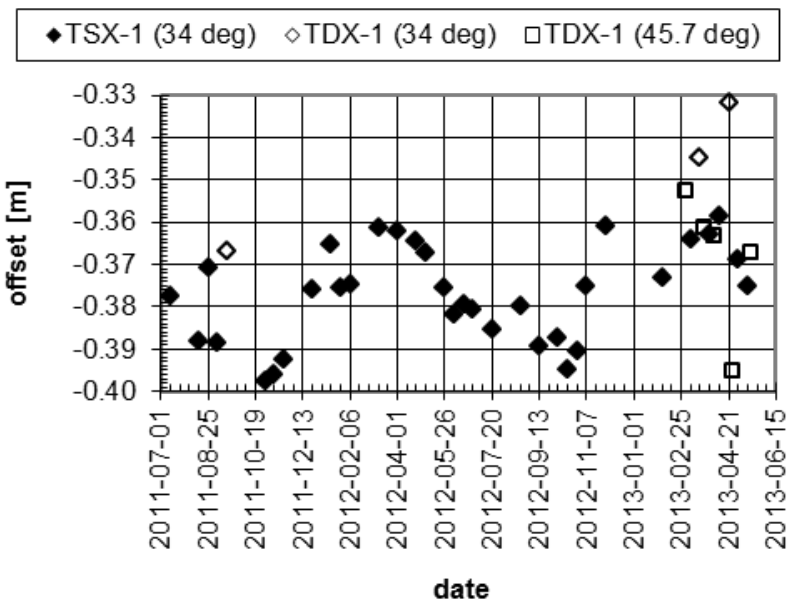

Figure 3: Temporal progression of the range offset

standard deviation for this satellite amounts to 11.3 millimeters in azimuth and 17.7 millimeters in range.

However, there is a strong temporal correlation between the measured range localization errors as evident in Figure 3. A visual inspection of the temporal progression reveals that a good portion of the standard deviation results from a slow variation. In contrast, almost all immediately neighboring measurement values differ by millimeters for datatakes acquired by the same sensor. Thus, on a shortterm scale, the measurements suggest that the localization accuracy for TSX-1 and TDX-1 could be further improved. This effect is subject to ongoing investigations. Comparing immediately neighboring data-takes from different sensors, there may be a range offset of approximately 2 centimeters between TSX-1 and TDX-1 datatakes where the TDX-1 datatakes are shifted more towards far range. However, as this observation is based on only 3 TDX-1 datatakes, it has to be verified with further measurements.

As the orientation of the corner reflector cannot be changed without invalidating the on-ground measurement of its phase center coordinates, it takes a second corner reflector to verify the transferability of the recent measurement results to datatakes with different orbit orientation or distinctly different elevation angle. In contrast, a moderate change in the acquisition geometry does not affect the visibility of the unchanged corner reflector even though its radar cross section is slightly reduced. Thus, we started a second measurement series where the Wettzell corner reflector is imaged from a neighboring orbit track. Compared to the primary measurement series the elevation angle changes from 34 to 45.7 degrees. The observed loss in radar cross section is approximately $2 \mathrm{~dB}$. At present the second measurement series consists of 5 datatakes recorded by TDX-1 between March 2, 2013 and May 18, 2013. Its localization results are included in Figures 2 and 3. As a first result based on the current limited number of recorded datatakes, the plots reveal that there is at least no coarse localization offset against the results of the primary measurement series. 


\section{CONCLUSIONS}

Both recent measurement series started on July 12, 2011 and March 2, 2013 respectively, will be continued. One major topic in the next stage of our project is the investigation of the angular dependency of our corrections. Within this context, a second corner reflector with a different orientation will be installed soon at the Wettzell test site and will enlarge the variability of usable acquisition geometries. Another major topic is the worldwide reproducibility of the achieved performance. This will be analyzed by the setup of comparable high precision test sites throughout the world - one additional test site with two corner reflectors was recently installed near DLR's O'Higgins receiving station on the Antarctic Peninsula. The setup of another test site near the GNSS reference station Metsähovi, Finland, is in progress.

In the long term, the geometric calibration of SAR sensors and the annotated corrections of SAR products will substantially benefit from the investigated correction schemes. Moreover, the substantially improved localization accuracy renders new SAR applications possible, e.g. in [20] we discussed the applicability of stereo SAR.

\section{ACKNOWLEDGMENTS}

The work was partially funded by the German Helmholtz Association HGF through its DLR@Uni Munich Aerospace project "Hochauflösende geodätische Erdbeobachtung". We thank the Federal Agency for Cartography and Geodesy (BKG) who kindly allowed us to install the corner reflectors at their Geodetic Observatory in Wettzell.

\section{REFERENCES}

[1] M. Eineder, C. Minet, P. Steigenberger, X.Y. Cong, and T. Fritz, "Imaging Geodesy - Toward Centimeter-Level Ranging Accuracy with TerraSAR-X," IEEE Transactions on Geoscience and Remote Sensing, vol. 49, no. 2, pp. 661-671, 2011.

[2] U. Balss, M. Eineder, T. Fritz, H. Breit, and C. Minet, "Techniques for High Accuracy Relative and Absolute Localization of TerraSAR-X / TanDEM-X Data," Proc. IGARSS 2011, Vancouver, pp. 2464-2467, 2011.

[3] A. Schubert, M. Jehle, D. Small, and E. Meier, "Mitigation of Atmosphere Perturbations and Solid Earth Movements in a TerraSAR-X Time-Series," Journal of Geodesy, Online First, September 23, 2011.

[4] A. Schubert, D. Small, M. Jehle, and E. Meier, "COSMOSkyMed, TerraSAR-X, and RADARSAT-2 Geolocation Accuracy after Compensation for Earth-System Effects," Proc. IGARSS 2012, Munich, pp. 3301-3304, 2012.

[5] X.Y. Cong, U. Balss, M. Eineder, and T. Fritz, "Imaging Geodesy - Centimeter-Level Ranging Accuracy with TerraSAR-X:
An Update", Geoscience and Remote Sensing Letters, vol. 9, no. 5, pp. 948-952, 2012.

[6] U. Balss, X.Y. Cong, R. Brcic, M. Rexer, C. Minet, H. Breit, M. Eineder, and T. Fritz, "High Precision Measurement on the Absolute Localization Accuracy of TerraSAR-X," Proc. IGARSS 2012, Munich, pp. 1625-1628, 2012.

[7] Cumming, I. G., and F. H. Wong, Digital Processing of Synthetic Aperture Radar Data: Algorithms and Implementations, Artech House, Boston, MA, 2005, ch. 4.2-4.3, pp. 114-129.

[8] Hofmann-Wellenhof, B., H. Lichtenegger, and E. Wasle, GNSS Global Navigation Satellite Systems, Springer, Wien, New York, 2008.

[9] Meindl, M., R. Dach, and Y. Jean (eds.), International GNSS Service Technical Report 2011, Astronom. Institute Univ. of Bern, 2012.

[10] J. Saastamoinen, "Contributions to the Theory of Atmospheric Refraction Part II. Refraction Corrections in Satellite Geodesy," Bulletin Géodésique, vol. 107, no. 1, pp. 13-34, 1973.

[11] J. Kouba, "Implementation and Testing of the Gridded Vienna Mapping Function 1 (VMF1)," Journal of Geodesy, vol. 82, no. 4-5, pp. 193-205, 2007

[12] J. Boehm, and H. Schuh, "Vienna Mapping Functions in VLBI Analyses," Geophys. Res. Lett., 31, L01603, doi: 10.1029/2003GL018984, 2004.

[13] Gisinger, C., Atmospheric Corrections for TerraSAR-X Derived from GNSS Observations, master thesis, TU Munich, 2012.

[14] Bilitza, D., Virtual Ionosphere, Thermosphere, Mesosphere Observatory (VITMO), Goddard Space Flight Center, Greenbelt, http://omniweb.gsfc.nasa.gov/vitmo/iri vitmo.html (20 Sep. 2012).

[15] Petit, G., and B. Luzum (eds.), IERS Conventions (2010), (IERS Technical Note 36), Verlag des Bundesamtes für Kartographie und Geodäsie, Frankfurt, 2010, ch. 7, pp. 99-122.

[16] Petrov, L., and J. Boy, Study of the Atmospheric Pressure Loading Signal in Very Long Baseline Interferometry Observations, Journal of Geophysical Research, 109 (B03405), 2007.

[17] Goddard Space Flight Center: http://lacerta.gsfc.nasa.gov/aplo_eph

[18] M. Schwerdt, B. Bräutigam, M. Bachmann, B. Döring, D. Schrank, and J.H. Gonzalez, "Final TerraSAR-X Calibration Results Based on Novel Efficient Methods," IEEE Transactions on Geoscience and Remote Sensing, vol. 48, no. 2, pp. 677-689, 2010.

[19] Fritz, T., and M. Eineder, TerraSAR-X Ground Segment Basic Product Specification Document, TX-GS-DD-3302, v1.5, 2008.

[20] U. Balss, C. Gisinger, X.Y. Cong, M. Eineder, R. Brcic, "Precise 2-D and 3-D Ground Target Localization with TerraSAR-X", Proc. ISPRS Hannover Workshop 2013 (to be published). 